\title{
Experimental Realization of Transverse Ising model on kagome and triangular lattice antiferromagnets \\ Zhiling Dun ${ }^{1}$ \\ ${ }^{1}$ No affiliation given \\ dunzhiling@gmail.com
}

Transverse Ising model on a frustrated lattice is expected to host intriguing quantum disordered states at low temperatures due to the combination of an ice-like magnetic degeneracy and quantum-tunneling terms. We demonstrate that these models can be realized experimentally on rare-earth-based antiferromagnets, specifically, on the tripod kagome magnet $\mathrm{Ho} 3 \mathrm{Mg} 2 \mathrm{Sb} 3 \mathrm{O} 14$, and the triangular lattice antiferromagnet $\mathrm{TmMgGaO} 4$. In both systems, Ising moments and intrinsic transverse fields originate from the crystal field of a non-Kramer's ion, whose magnitudes can be determined by an effective point charge analysis of the crystal field excitations [1]. Using neutron scattering, magnetic susceptibility, and thermodynamic measurements, (i) in $\mathrm{Ho} 3 \mathrm{Mg} 2 \mathrm{Sb} 3 \mathrm{O} 14$, we observe a symmetry-breaking transition at $0.32 \mathrm{~K}$ to a partially ordered state which is characterized by a fragmentation of the magnetic moments and persistent inelastic magnetic excitations [2]; (ii) in $\mathrm{TmMgGaO}$, we found evidence for the existence of an intermediate Kosterlitz-Thouless phase between $0.9 \mathrm{~K}$ and $5 \mathrm{~K}$ which is characterized by short-range magnetic correlations and binding/unbinding of spin vortex-antivortex pair [3]. Our results point out a practical option to introduce quantum fluctuations in frustrated magnets and call for further experimental explorations of quantum magnets with short-range magnetic order.
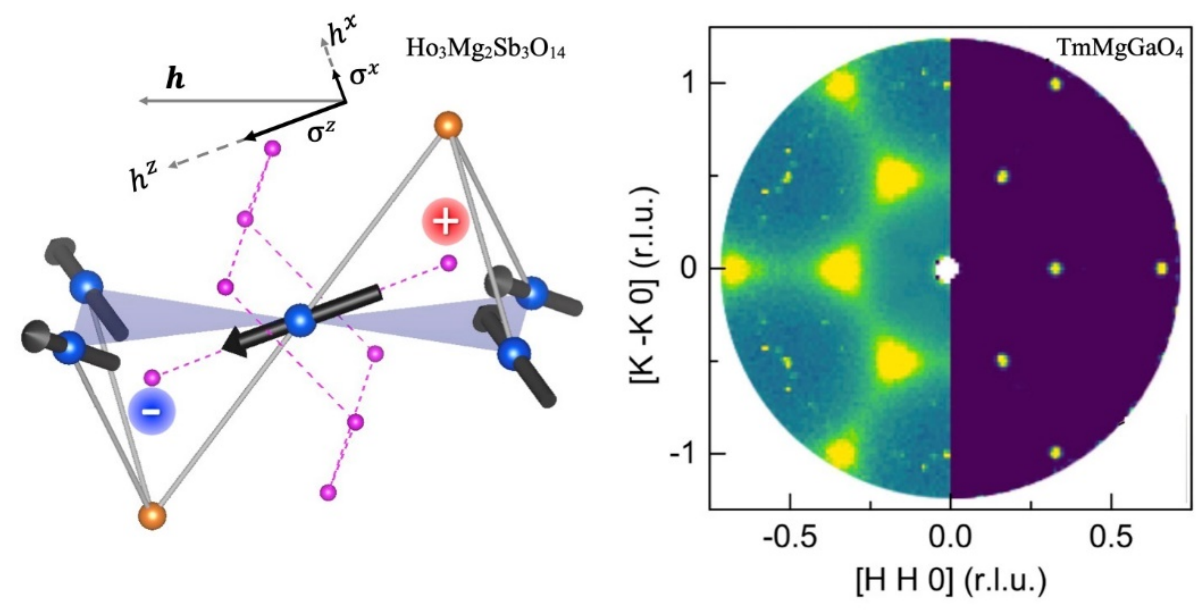

Figure 1 